\title{
Don et sciences sociales. Théories et pratiques
} croisées (compte rendu)

Centre d'études médiévales d'Auxerre, 27-28 janvier 2006

\section{Eliana Magnani}

\section{(2) OpenEdition}

\section{Journals}

Édition électronique

URL : https://journals.openedition.org/cem/383

DOI : $10.4000 /$ cem.383

ISSN : 1954-3093

Éditeur

Centre d'études médiévales Saint-Germain d'Auxerre

Édition imprimée

Date de publication : 15 août 2006

ISSN : 1623-5770

\section{Référence électronique}

Eliana Magnani, « Don et sciences sociales. Théories et pratiques croisées (compte rendu) », Bulletin du centre d'études médiévales d'Auxerre | BUCEMA [En ligne], 10 | 2006, mis en ligne le 08 septembre 2006, consulté le 22 septembre 2022. URL : http://journals.openedition.org/cem/383 ; DOI : https:// doi.org/10.4000/cem.383

Ce document a été généré automatiquement le 22 septembre 2022.

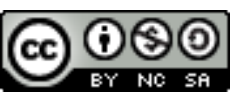

Creative Commons - Attribution - Pas d'Utilisation Commerciale - Partage dans les Mêmes Conditions 4.0 International - CC BY-NC-SA 4.0

https://creativecommons.org/licenses/by-nc-sa/4.0/ 


\title{
Don et sciences sociales. Théories et pratiques croisées (compte rendu)
}

\author{
Centre d'études médiévales d'Auxerre, 27-28 janvier 2006
}

\section{Eliana Magnani}

1 La théorie que Marcel Mauss exposait dans son «Essai sur le don. Forme et raison de l'échange dans les sociétés archaïques ", en $1924^{1}$, est devenue l'une des théories les plus utilisées et discutées en anthropologie. Depuis les années 1980, elle a exercé, et continue à exercer, une indéniable fascination dans d'autres disciplines. La question du don a ainsi été intégrée dans le répertoire des notions utilisées par différentes disciplines en sciences sociales, orientant leurs intérêts, souvent sans aucune discussion préalable sur sa pertinence. Il n'empêche que dans certains domaines, ce phénomène d'incorporation irraisonné était un passage obligé, voire bénéfique, qui a permis d'ouvrir des questionnaires et d'alimenter de nouvelles réflexions. La table ronde qui s'est tenue à Auxerre les 27 et 28 janvier 2006, partait de ces constatations pour ouvrir une large discussion sur le don, entre historiens, archéologues, anthropologues, sociologues et philosophes. Il s'agissait, à l'initiative des historiens médiévistes, de porter un regard croisé sur les pratiques et les méthodes, d'évaluer les interprétations et les modèles mis en jeu. Les interventions qui ont servi de base aux discussions ont été de trois sortes: des panoramas critiques, des études de cas particuliers et des exposés à portée théorique.

2 L'imprégnation de la notion maussienne de don/contre-don, employée souvent de façon mécanique et réductrice dans la médiévistique depuis Georges Duby (1973) et les importants travaux en histoire sociale des médiévistes nord-américains des années 1980 (Patrick Geary, Stephen White, Barbara Rosenwein...), a été traitée par Eliana MAGNANI. Se référant à Mauss de manière tronquée, et donnant ainsi une coloration anthropologique à leurs interprétations, les médiévistes ignorent souvent que le principe de réciprocité inhérent à la notion de «don-échange» (Gebentausch, Geschenktausch) apparaît dès le milieu du XIxe siècle chez les historiens allemands du droit germanique. Jacob Grimm est le premier, dès 1848, à lancer l'idée du contre-don (Gegengabe), dans une étude à caractère étymologique «sur faire des cadeaux et des 
dons» («Über Schenken und Geben»). Quand Mauss écrit «l'Essai sur le don», la notion de don contre-don avait déjà une longue histoire qu'il connaissait probablement. Dans un article de 1921 sur «Une forme ancienne de contrat chez les Thraces », Mauss termine en souhaitant que des spécialistes réalisent l'étude "de la donation germanique et des échanges dans les textes celtiques». Il espérait qu'on y trouve, comme il venait de le faire pour quelques textes grecs d'Homère, Xénophon, Thucydide et Anaxandride, "des formes de traité, de mariage, d'échange, de prestations religieuses et esthétiques mêlées à peu près comme en Mélanésie ou en Amérique ».

3 Son proche collaborateur Henri Hubert, sociologue des religions et archéologue, a été le premier à y appliquer la théorie du don aux sociétés celtiques. Hubert fonde cependant son analyse sur les légendes et mythes irlandais et gallois connus par leur version écrite seulement à partir du $\mathrm{X}^{\mathrm{e}}$ siècle de notre ère, et néglige les sources archéologiques et les témoignages des auteurs classiques sur les Celtes. Comme l'a exposé Luc BARAY, la mort prématurée d'Henri Hubert ( $†$ 1927), associée à un rejet de l'archéologie pour tout ce qui est théorique, ont fait que le concept du don n'est à nouveau employé dans les analyses des sociétés protohistoriques qu'en 1978, avec les travaux de Susan Frankenstein et Michael Rowlands, sur le système princier du premier âge du Fer, repris et étendu aussi à l'âge du Bronze en 1987, par Patrice Brun dans son Princes et princesses de la Celtique. Alors que Frankenstein-Rowlands utilisent plutôt le concept d'économie de biens de prestige, $\mathrm{P}$. Brun se réfère explicitement à la théorie maussienne du don et à la notion d'« économie-monde» de Fernand Braudel. En retenant les formes agonistiques du don (potlatch) et en appliquant indistinctement aux sociétés celtiques le modèle dégagé pour les royautés africaines par Claude Meillassoux $(1960,1972)$, les analyses proposées par ces auteurs pêchent, entre autres, par la confusion grandissante établie entre les concepts de "système de don » et d'« économie de biens de prestige ", pourtant bien distincts. Ils diluent ainsi la valeur heuristique que le don pourrait avoir dans la compréhension de ces sociétés, représentées par eux comme des sociétés toujours en crise, instables et dépendantes de l'économie-monde.

4 Pour ce qui est de la Grèce ancienne, présentée par Évelyne scheID, l'essai de Mauss de 1921 sur les «festins » chez les Thraces, ne suscite pas d'échos jusqu'aux articles précurseurs d'Émile Benveniste sur "Le don et l'échange dans le vocabulaire indoeuropéen ", et de Louis Gernet sur "La notion mythique de la valeur en Grèce », les deux de 1948. Ils restent cependant méconnus jusqu'au milieu ou la fin des années 1960. C'est à cette époque que l'ouvrage de Moses I. Finley, Le monde d'Ulysse, de 1954, très influencé par Karl Polanyi, est traduit en français. Avec Finley s'impose l'idée du don comme lieu de toutes les institutions de la Grèce archaïque. Plus largement, c'est par son biais que la notion de don contre-don entre dans l'historiographie en général. En fait, le recours au don pour caractériser la société homérique, mais aussi les sociétés celtes ou la société du haut Moyen Âge, sert désormais d'élément classificatoire pour ceux qui, dans une perspective évolutionniste ou comparatiste, les considèrent comme des sociétés « primitives ». C'est une autre perspective qu'adopte Paul Veyne (1976), en étudiant l'évergétisme ancien, cherchant à bien le distinguer, au Bas Empire, de la donation chrétienne. Le don ne reste pas moins l'une des clés d'interprétation de la société grecque, comme dans certains épisodes qui précèdent les guerres médiques 
rapportés par Hérodote, où les échanges entre acteurs de statuts différents, le roi et ses sujets, par exemple, peuvent être interprétés en termes de " réciprocité inégale ».

5 Une autre approche historienne, présentée par Jean-Paul DESAIVE à partir de la documentation auxerroise des XVII ${ }^{\mathrm{e}}$ et XVIII ${ }^{\mathrm{e}}$ siècles, consiste à déceler les formes du don dans les modes de transmission patrimoniale, tout au long du cycle de vie. Au moment de l'union matrimoniale, le contrat de mariage, qui prévoit la succession lors de la disparition de l'un des conjoints ; la démission des biens en contrepartie du soutien au moment de la vieillesse et de la maladie ; la donation entre vifs, surtout entre les époux sans enfant; et le testament, dont la dimension spirituelle reste la première motivation, mais qui fait une large place aux récompenses adressées aux serviteurs domestiques, tout en préservant le patrimoine des héritiers directs.

6 Le détour chez les Miraña de l'Amazonie colombienne, a permis à Dimitri KARADIMAS d'évoquer le don dans un contexte interprétatif dominé par le paradigme anthropologique de la "prédation" (Eduardo Viveiros de Castro). L'analyse du rituel des esprits des animaux, où les humains invitent les «maîtres des animaux » en vue d'être accueillis ensuite dans la forêt, fait apparaître le don comme moyen de légitimation de l'acte de prédation: on donne, on n'attend pas de contrepartie, on prend. Au modèle du "don-échange » - donner, recevoir, rendre, où le donataire reçoit et rend - il est possible alors d'opposer le modèle du "don-prédation " - donner, recevoir, prendre, où le donateur donne et prend, dans un circuit où donner n'est pas la garantie de l'alliance et où le conflit peut surgir à tout moment. Une telle perspective invite à nuancer la notion globalisante de prédation pour caractériser les sociétés amazoniennes, en prenant en considération des phénomènes qui renvoient à la réciprocité.

7 Dans toutes ces approches se pose le problème des notions mises en œuvre dans les interprétations historiques, archéologiques et anthropologiques, et plus généralement la place de ces disciplines dans l'élaboration des concepts, surtout dans une situation "post-moderne» de "crise» des sciences sociales, avec l'abandon de l'idée d'une théorie sociologique générale, au profit d'un investissement dans la description de la diversité sociale. Ilana SILBER a évoqué ainsi la question des outils conceptuels souples qui pourraient rendre compte du don dans sa très grande complexité, sans tomber dans une typologie à outrance où il se diluerait en tant que catégorie distincte. Elle propose la métaphore sociologique de "répertoire " (Michèle Lamont, Ann Swidler, Laurent Thévenot) dont la structuration interne pourrait conduire à terme, selon le contexte historique et social précis, à la définition d'un "champs» (Pierre Bourdieu, sans toutefois, adhérer à tout son cadre théorique). À titre d'exemple, une typologie de départ issue de la sociologie comparée du don dans les religions (y compris les donations aux monastères au Moyen Âge), donnerait une liste à quatre termes identifiant les alternatives structurantes du répertoire du don dans ce genre de contexte : les dons aux dieux (dont le sacrifice, mais pas seulement), aux pauvres, aux institutions et élites religieuses (sacerdotales/sacramentelles), et à la cité. Cela permet également de souligner l'intérêt de la notion de «registre » du don telle qu'elle a été développée par Natalie Zemon Davies dans ses travaux sur la France du $\mathrm{XvI}^{\mathrm{e}}$ siècle, exemple bien précis de répertoire du don, avec ses composantes et structures de champs spécifiques.

8 Il reste, cependant, à évaluer le legs de Mauss et de sa « découverte » du don - sorte d'hybride, mêlant intérêt et désintéressement, obligation et liberté - à déterminer 
l'effectivité ou pas de son universalité et de sa charge normative. Considérant le don comme une "réalité spécifique " qui peut être expliquée par elle-même, propre à résoudre les paradoxes de l'existence humaine, Alain caILlé propose l'investigation empirique - dans son cas sur ce qui reste du don dans la société contemporaine comme moyen d'organiser un système de questions en vue d'une théorie sociologique générale. Le don s'articulerait autour de quatre pôles interdépendants mais irréductibles entre eux, en tant que concept politique (l'alliance plutôt que la guerre, rivalité), vecteur des relations humaines (l'intérêt pour soi et pour autrui, l'aimance, l'altérité), obligation (durkheimienne) et part de liberté et de créativité. Une telle proposition va à l'encontre des interprétations du don de type "économiciste " (autour de l'idée de " profit », dont l'approche de P. Bourdieu), ou « inexistentialistes » (le don n'existe pas - comme pour certains philosophes tel Jacques Derrida-, ou dans très peu de sociétés), ou qui considèrent le don comme " phénomène secondaire » (par exemple, la religion serait plus importante, d'après Maurice Godelier).

Le parti pris théorique exposé par Alain TESTART emprunte une autre direction, critique en relation à la théorie maussienne qui, en insistant sur l'obligation du contredon, aurait confondu don et échange (dans l'interprétation de la Kula, par exemple). Faisant jouer des catégories juridiques, A. Testart distingue nettement trois formes de transfert - le don, l'échange et l'impôt - à partir de la notion de contrepartie (ce qui vient en retour d'un transfert), en tant que phénomènes opposés. La contrepartie dans l'échange est exigible et obligatoire, alors qu'elle ne l'est pas dans le don. Le don est par principe sans condition. Même si on peut attendre, demander ou solliciter un retour (un contre-don), on ne peut pas l'exiger. Le don ainsi défini, plutôt qu'un phénomène universel, serait une forme sociale qui peut assumer des expressions différentes dans des sociétés différentes.

10 L'universalité du don est justement l'hypothèse testée par la réflexion philosophique proposée par François ATHANÉ, à partir d'une approche naturalistecomportementaliste associée au rapport à une règle et au langage, acte performatif et déclaratif qui implique le changement du statut déontique des objets. Si le don est un fait universel humain, il faut vérifier s'il est ailleurs dans la nature et se référer notamment aux travaux en éthologie et à la notion d'altruisme chez les animaux. La transmission des gènes ne passe pas seulement par la reproduction sexuée, mais aussi par un comportement altruiste qui favorise la reproduction d'individus apparentés (théorie de la sélection de la parentèle, William D. Hamilton -1964). Dans le transfert de ressources dans l'espèce humaine, il y a une part de naturalité, comme il y a une part de culture, mais le comportement ne peut suffire pour définir le don, d'où le besoin du rapport à une règle. Si le don est universel, il est lié à l'universalité d'une règle (loi), qui pourrait être le «don nourricier » des adultes vers le nourrisson, forme première de transfert de ressources.

11 La diversité des approches présentées au cours de cette table ronde et les vives et riches discussions qu'elles ont suscitées, ont laissé transparaître la difficulté du dialogue entre les différentes disciplines, mais aussi tout son intérêt. Comme l'a noté Anita GUERREAU-JALABERT dans les considérations finales de la rencontre, les problèmes de communication entre chercheurs résident en grande partie dans l'ignorance de l'évolution de la civilisation occidentale et des notions qui se sont imposées à nous. Il reste à mener une large réflexion sur ces notions, pas seulement sur le don, pour lesquelles l'historien médiéviste, s'occupant de la société qui a précédé la nôtre, se 
trouve bien placé. L'exemplum médiéval montre l'articulation entre l'Ecclesia, institution globalisante, et le christianisme (auquel il ne faut pas plaquer la notion moderne de «religion»), et l'étroite imbrication entre le système social et le système de représentations. Dans cette société référée au divin, le lien social se représente en termes de caritas, dans le «sens de l'amour spirituel, véhiculé par le Saint-Esprit et transitant par Dieu » et qui irrigue toute la société. La circulation des biens étant l'un des moyens à travers lequel on établit et renouvelle des liens sociaux, c'est à l'intérieur de cette logique de "circulation généralisée » de la caritas, que les modalités pratiques du don sont comprises au Moyen Âge.

\section{Rappel du programme :}

- Eliana MAGNANI (CNRS-Auxerre/Dijon), Les médiévistes et le don, ou l'imprégnation du modèle maussien.

- Luc BARAY (CNRS-Sens/Dijon), Le don et la protohistoire : du bon usage d'un concept anthropologique

- Ilana SILBER (univ. Bar-Ilan), Registe(s) et répertoire(s) du don : avec mais aussi après Mauss?

- Alain CAILlé (univ. Paris X), Ce que l'on appelle si mal le don... Que le don est de l'ordre du don malgré tout.

- Jean-Paul DESAIVE (EHESS - Paris), Le testament féminin en Auxerrois sous l'Ancien Régime : un révélateur de solidarités plus spécifiquement féminines?

-Évelyne SCHEID (univ. Paris 13), Une réciprocité inégale. L'usage politique du don entre Grecs et Perses à la veille des guerres médiques selon Hérodote.

- Alain TESTART (CNRS-Paris), Définition du don - et pourquoi la kula n'est pas du don

- Dimitri KARADIMAS (CNRS-Paris), Le don ou le droit à la prédation : le rituel des esprits des animaux chez les Miraña d'Amazonie colombienne

- François ATHANÉ (univ. Paris X), Le don : un point de vue philosophique

- Anita guerreau-JAlabert (École nationale des Chartes), Conclusions.

\section{NOTES DE FIN}

1. La plupart des travaux de Marcel Mauss sont accessibles en ligne à cette adresse : http://classiques.uqac.ca/classiques/mauss_marcel/mauss_marcel.html

\section{INDEX}

Mots-clés : sciences sociales, don 